\title{
Radiative cascade from quantum dot metastable spin-blockaded biexciton
}

\author{
Y. Kodriano, ${ }^{1}$ E. Poem, ${ }^{1}$ N. H. Lindner,${ }^{2}$ C. Tradonsky, ${ }^{1}$ B. D. Gerardot, ${ }^{3}$ P. M. Petroff,${ }^{4}$ J. E. Avron,,${ }^{1}$ and D. Gershoni ${ }^{1}$ \\ ${ }^{1}$ Department of Physics, The Technion-Israel Institute of Technology, Haifa 32000, Israel \\ ${ }^{2}$ Institute of Quantum Information, Caltech, Pasadena, California 91125, USA \\ ${ }^{3}$ School of Engineering and Physical Sciences, Heriot-Watt University, Edinburgh EH14 4AS, United Kingdom \\ ${ }^{4}$ Materials Department, University of California, Santa Barbara, California 93106, USA
}

(Received 1 July 2010; revised manuscript received 2 September 2010; published 29 October 2010)

\begin{abstract}
We detect a radiative cascade which initiates from a metastable biexciton state in a neutral semiconductor quantum dot. In this biexciton, the heavy holes form a spin-triplet configuration, Pauli blockaded from relaxation to the spin-singlet ground state. The triplet biexciton has two photon-phonon-photon decay paths. Unlike in the singlet-ground-state biexciton radiative cascade, in which the two photons are colinearly polarized, in the triplet-biexciton cascade they are cross-linearly polarized. We measured the two-photon polarization density matrix and show that the phonon emitted when the intermediate exciton relaxes from excited to ground state, preserves the exciton's spin. The phonon, thus, does not carry with it any which-path information other than its energy. Nevertheless, entanglement distillation by spectral filtering was found to be rather ineffective for this cascade. This deficiency results from the opposite sign of the anisotropic electron-hole exchange interaction in the excited exciton relative to that in the ground exciton.
\end{abstract}

DOI: 10.1103/PhysRevB.82.155329

PACS number(s): 78.67.Hc, 73.21.La, 78.47.jd

\section{INTRODUCTION}

A quantum dot (QD) containing two electron-hole (e-h) pairs-a biexciton, returns to its vacuum state by emitting two photons in a radiative cascade. The radiative cascade mostly discussed in the literature ${ }^{1-7}$ initiates from the (nondegenerate) ground state of the biexciton in which the two electrons and two holes form spin singlets in their respective lowest single carrier energy levels. The QD relaxes to its vacuum state by spontaneously emitting two photons. The emission of the first photon brings the system into the manifold of intermediate single exciton states. Two out of the four possible single exciton states are optically active (bright). If these two "bright exciton" states were energetically degenerate, the two emitted photons would have been polarization entangled. ${ }^{1,5-7}$ Usually, however, due to the reduced symmetry of the QD, the bright exciton states are not degenerate. ${ }^{8-10}$ This removes the radiative cascade's "whichpath" ambiguity. Consequently, the polarization state of the emitted photon pair contains mostly classical correlations, ${ }^{2,3}$ and only a very small, usually undetectable, degree of entanglement. The degree of entanglement can be increased to a measurable level by spectrally filtering out most of the unentangled photon pairs (those which are only classically correlated) while keeping photon pairs for which which-path ambiguity exists. ${ }^{4,11,12}$

Here, we report on the observation of a radiative cascade, initiating from a metastable, spin-blockaded biexcitonic state. This biexciton state is composed of two electrons in their ground state, and two heavy holes, one in its ground state and one in an excited state, forming a spin triplet. Since this biexciton state is spin blockaded from relaxation to the ground biexciton state, the recombination of a ground level e-h pair occurs first.

When this happens, a single exciton which contains a hole in an excited state is left in the QD. The hole alone is no longer spin blockaded, and it quickly relaxes to its ground state, releasing its excess energy by emitting a phonon. The ground-state exciton thus formed, spontaneously recombines radiatively, and the QD is left in its vacuum state. Consequently, the entire process involves the cascaded emission of two photons and one phonon. The intermediate nonradiative decay is fast (few tens of picosecond ${ }^{13}$ ), and as we show below, preserves the exciton's spin.

For a spin-preserving nonradiative decay, one expects all the which-path information carried by the intermediate phonon to reside in its energy. Therefore, one would naively expect spectral filtering which chooses the cases where the phonons have the same energy, to be effective in restoring entanglement also in this cascade.

We show below that this is not the case. We applied the same filtering scheme for the two types of radiative cascades, from the same quantum dot, under the same conditions. However, whereas for the ground-state, singlet-biexciton cascade, entanglement was restored, it was not restored for the triplet, spin-blockaded, cascade. In Sec. V below, we show that the opposite sign in the fine-structure splitting of the two intermediate excitonic levels, combined with the fluctuating electrostatic environment (spectral diffusion), ${ }^{12}$ is responsible for this deficiency.

\section{ENERGY LEVELS}

The biexciton states which we discuss here are composed of two electrons in their ground state, and two heavy holes, one in its ground state and one in an excited state. Four different spin configurations are thus possible for the two holes, as illustrated in Fig. 1. Three of them are symmetric under particle exchange (triplet) and one is antisymmetric (singlet). The triplet states are energetically lower than the singlet state due to the hole-hole exchange interaction [typically about $4 \mathrm{meV}$ in our QDs (Ref. 13)].

Since the ground biexciton state is formed by two holes in the same level, by Pauli's exclusion principle, they must also 


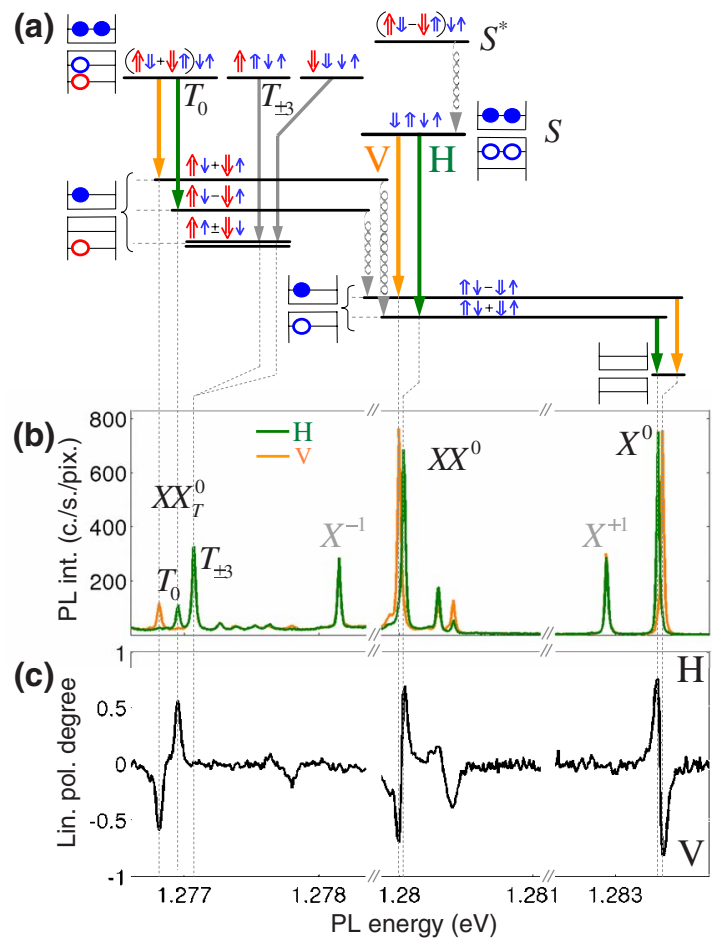

FIG. 1. (Color online) (a) Energy levels diagram for excitons and biexcitons in a neutral QD. Single-carrier level occupations are given along side each many-carrier level. The spin wave functions are depicted above each level. The symbol $\uparrow(\Downarrow)$ represents spin-up (spin-down) electron (hole). Short blue (long red) symbols represent charge carriers in the first (second) energy level. S $\left(\mathrm{S}^{*}\right)$ indicates ground (excited) biexciton hole-singlet state. $\mathrm{T}_{0}\left(\mathrm{~T}_{ \pm 3}\right)$ indicates the metastable spin-triplet-biexciton state with $z$-axis spin projection of $0( \pm 3)$. The solid (curly) vertical arrows indicate spin preserving (non)radiative transitions. Green (dark-gray) [orange (light-gray)] arrows represent photon emission in horizontal- $\mathrm{H}$ (vertical-V) polarization. (b) Polarized PL spectra. H (V) in green (orange). Spectral lines which are relevant to this work are marked and linked to the transitions in (a) by dashed lines. (c) Linear polarization spectrum. The value $1(-1)$ means full $\mathrm{H}(\mathrm{V})$ polarization.

be in an antisymmetric spin configuration. Relaxation from the excited hole-singlet-biexciton state into the ground biexciton state does not require a change in the spin configuration of the two holes. The energy mismatch between the two states is taken by generating a single acoustic phonon. This process occurs within a few tens of picosecond, ${ }^{13}$ orders of magnitude faster than the radiative lifetime in these QDs [about $1 \mathrm{~ns}$ (Ref. 14)]. In contrast, the relaxation from the hole-triplet-biexciton states does require a change in the spin configuration of the two heavy holes. Since these changes are slower than the radiative rate, these metastable states are "spin blockaded" from nonraditive phonon-assisted relaxation. ${ }^{13}$ It follows that while hole-triplet-biexciton states can radiatively decay before they relax nonradiatively, the excited hole-singlet-biexciton state cannot. Therefore, we did not observe emission from this state and it will not be discussed here further.

A diagram of the relevant energy levels of a neutral QD is presented in Fig. 1(a). The ground (excited) singlet-biexciton state is marked by $\mathrm{S}\left(\mathrm{S}^{*}\right)$. The metastable, triplet-biexciton states are marked by $\mathrm{T}_{0}$ and $\mathrm{T}_{ \pm 3}$, where the subscripts stand for the total two holes' spin projection on the QD's growth direction. Since the electrons form a spin singlet in their ground state, the electron-hole exchange interaction vanishes, and the triplet states remain degenerate. Hole-hole anisotropic exchange interaction is expected to remove this degeneracy ${ }^{15}$ by lowering the $T_{ \pm 3}$ states with respect to the $\mathrm{T}_{0}$ state. This separation, in our case is smaller than the isotropic electron-hole exchange interaction, which removes the degeneracy between the dark and bright single exciton states. Therefore, the energy order between the $\mathrm{T}_{0}$ and $\mathrm{T}_{ \pm 3}$ biexcitonic emission lines is the same as if there was no hole-hole anisotropic exchange interaction, and we safely leave it out in the following discussion.

Recombinations from the $\mathrm{T}_{ \pm 3}$ states lead to the optically inactive (dark) exciton intermediate states [see the spectral line in Fig. 1(b)]. Radiative decay does not proceed from these states and they are discussed elsewhere. ${ }^{16}$

The ground state, singlet biexciton recombines through one of the two single bright exciton states. Due to in-plane anisotropy, the eigenstates of the bright exciton are the symmetric and antisymmetric combinations of its $J_{z}= \pm 1$ spinprojection states ${ }^{10}$ [see Fig. 1(a)]. Radiative recombinations to these states and from them are thus colinearly polarized, as shown in Fig. 1(a), resulting in colinear polarization correlations between the cascading two photons. ${ }^{2,4}$

Similarly, the $\mathrm{T}_{0}$ biexciton state recombines to form an excited bright exciton in which the heavy hole resides in its second energy level. The eigenstates of this exciton are also the symmetric and antisymmetric combinations of spinprojection states. Here however, the energy order of the symmetric and antisymmetric eigenstates is opposite to that of the ground exciton. The reason for this order reversal is the difference between the spatial symmetry of the ground and the excited hole wave function. The anisotropic e-h exchange interaction, which induces this degeneracy removal, is proportional to the quadrupole moment between the electron and hole wave functions. ${ }^{14,17,18}$ Therefore there is a sign difference in the e-h exchange between the case in which the hole has $s$-like symmetry (ground-state exciton) and the case in which the hole has $p$-like symmetry (excited exciton). ${ }^{14,17}$

There is yet another marked difference between the spin blockaded, $\mathrm{T}_{0}$ biexcitonic transition and the ground state, $\mathrm{S}$ one. The difference is in the polarization selection rules for optical transitions. In the ground-state biexciton, the two holes form a spin singlet while in the $\mathrm{T}_{0}$ one they form a triplet. Therefore, while in the S-biexciton recombination, emission of horizontally (vertically) polarized photon results in symmetric (antisymmetric) bright exciton eigenstate, in the $\mathrm{T}_{0}$-biexciton recombination it results in antisymmetric (symmetric) eigenstate of the excited bright exciton. A similar effect happens in the recombination of a doubly charged exciton, ${ }^{14,19}$ there it is due to the final states.

Thus, the energy order of the biexciton doublet is the same for both biexcitons. This results from the fact that the difference in polarization selection rules is compensated by the sign difference in the anisotropic exchange interaction. This is indeed what we observe experimentally, as can be clearly seen in the polarized photoluminescence (PL) spectra presented in Fig. 1(b), and in the corresponding spectrum of 
linear polarization degree shown in Fig. 1(c).

The hole of the excited exciton states is not spin blockaded and it quickly relaxes (nonradiatively) to its ground state. If during this relaxation, the exciton's spin state is preserved, horizontally $(H)$ polarized $T_{0}$ biexciton recombination will be followed by vertically $(\mathrm{V})$ polarized ground-state exciton recombination [see Fig. 1(a)]. Thus, for the case of spin-preserving nonradiative relaxation, the photon pair emitted during the $\mathrm{T}_{0}$ biexciton cascade should be crosslinearly polarized, unlike the colinearly polarized photon pair emitted in the $\mathrm{S}$ biexciton cascade. Below we show that this is indeed the case.

\section{EXPERIMENTAL}

The studied sample was grown by molecular-beam epitaxy on a (001) oriented GaAs substrate. One layer of straininduced InGaAs QDs was deposited in the center of a 285$\mathrm{nm}$-thick intrinsic GaAs layer. The layer was placed between two distributed Bragg reflecting mirrors, made of 25 (bottom) and 10 (top) periods of pairs of $\mathrm{AlAs} / \mathrm{GaAs}$ quarter wavelength thick layers. This constitutes a one optical wavelength (in matter) microcavity for light emitted due to recombination of QD confined e-h pairs in their lowest energy levels. The microcavity increases light harvesting efficiency, for emission which resonates with its cavity mode.

For the optical measurements the sample was placed inside a tube immersed in liquid helium, maintaining sample temperature of $4.2 \mathrm{~K}$. A $\times 60,0.85$ numerical aperture, in situ microscope objective was used both to focus the exciting beam on the sample surface and to collect the emitted light. The collected light was split by a nonpolarizing beam splitter, and each beam was dispersed by a $1 \mathrm{~m}$ monochromator and detected by either an electrically cooled charge coupled device array detector or by a single channel, single photon, silicon avalanche photodetector. The system provides spectral resolution of about $10 \mu \mathrm{eV}$.

The polarization of the emitted light in each beam was analyzed using two computer-controlled liquid-crystal variable retarders and a linear polarizer. The retarders were carefully calibrated at the emission wavelength such that crosstalks between various polarization projections never exceeded $5 \%$.

Standard photon counting electronics was then used to measure the differences between the arrival times of two photons originating from two different spectral lines, at given polarizations. The histogram of these times, when normalized, gives the time-resolved intensity correlation function. The response function of the system and its temporal resolution $(\sim 0.4 \mathrm{~ns})$ were determined by measuring picosecond laser pulses. ${ }^{3,4}$

\section{RESULTS}

In Fig. 2(a) [Fig. 2(c)] we present measured time-resolved intensity correlation functions between the $\mathrm{T}_{0}[\mathrm{~S}]$ biexciton line and the exciton line, in both co-(dark gray, blue online) and cross-(light gray, red online) linear polarizations. It is clearly seen that while for the cascade starting from the $\mathrm{S}$
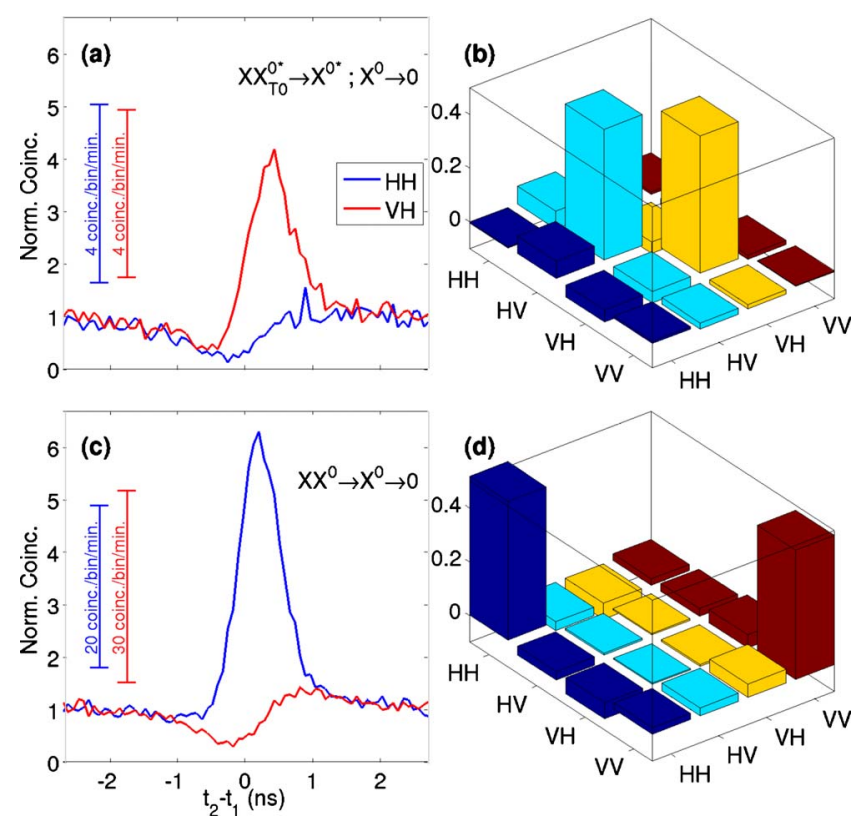

FIG. 2. (Color online) [(a) and (c)] Measured intensity correlation functions for the spin-blockaded and ground-state biexciton cascades, respectively. Blue (dark-gray) [red (light-gray)] line represents the correlation in co-[cross-]linear polarizations. The coincidence rates are indicated by the scale bars in units of coincidences per time bin (80 ps) per minute. [(b) and (d)] Real parts of the two-photon polarization density matrices measured for the spinblockaded and ground-state biexciton cascades, respectively.

biexciton, the emission of the exciton is "bunched" for colinear polarizations and "antibunched" for cross-linear polarizations, the exact opposite happens for the cascade starting from the $\mathrm{T}_{0}$ biexciton. This confirms our understanding of the $\mathrm{T}_{0}$ biexciton cascade. Moreover, our observations unambiguously demonstrate that there is no change in the spin state of the excited bright exciton during its phonon-assisted relaxation to the ground exciton state.

This important observation is not at all surprising. It agrees well with the suppressed heavy-hole-light-hole mixing in flat, strain-induced InAs/GaAs QDs. ${ }^{20}$ This mixing could have otherwise provided a channel for phonon-assisted spin nonpreserving relaxation. ${ }^{21}$ Another source of such relaxation is attributed to two-phonon processes mediated by the spin-orbit interaction. ${ }^{22}$ However, these processes are negligible in respect to interlevel, spin-preserving single phonon processes ${ }^{18}$ most relevant to the present case.

In addition, we performed full polarization tomography for both cascades, with and without spectral filtering., ${ }^{4,11,12}$ The resulting two-photon polarization density matrices for the case of no filtering are presented in Fig. 2(b) [Fig. 2(d)] for the $\mathrm{T}_{0}[\mathrm{~S}]$ biexciton cascade. Since the imaginary parts of the matrices were zero to within the accuracy of the measurement, only the real parts are displayed.

In Fig. 3 we present the polarization density matrices obtained for the case when spectral filtering is applied. While entanglement is restored for photon pairs emitted from the $\mathrm{S}$ biexciton cascade ${ }^{4}$ [Fig. 3(a)], no such restoration is observed for the $T_{0}$ cascade [Fig. 3(b)]. The reason for this difference is not the emission of an additional phonon in the 
(a)

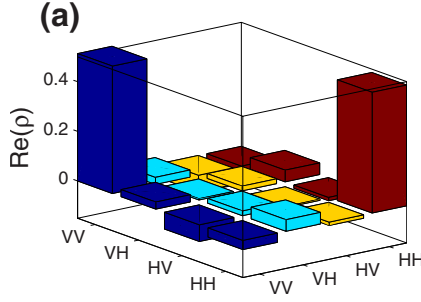

(b)
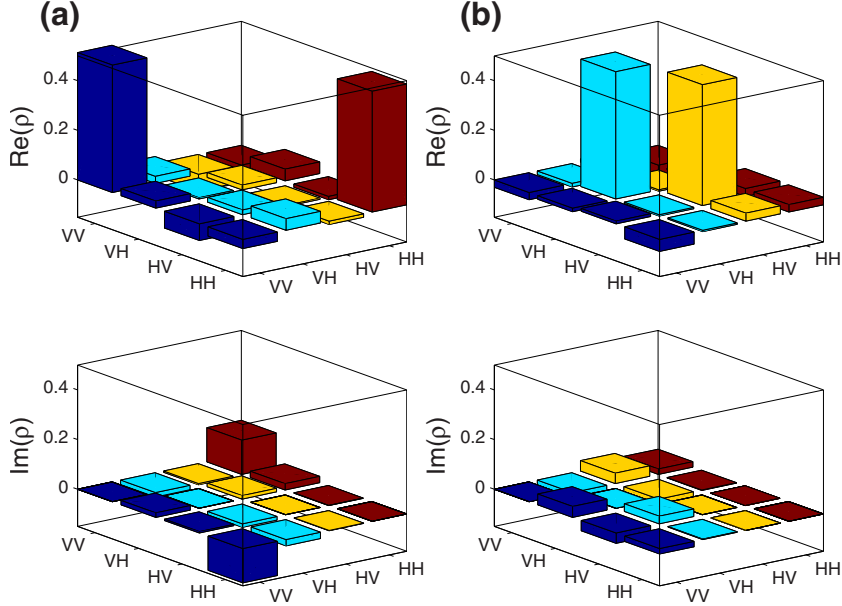

FIG. 3. (Color online) [(a) and (b)] Measured two-photon polarization density matrices for the ground-state $(\mathrm{S})$ and spin-blockaded $\left(\mathrm{T}_{0}\right)$ biexciton cascades, respectively, as obtained with spectral filtering. Real (imaginary) parts are shown in the top (bottom) panels. The Peres criterion (negativity of the partially transposed matrix) for the matrix in (a) $[(b)]$ is $0.15 \pm 0.03[0.05 \pm 0.1]$.

latter cascade, as one would naively assume. The emitted phonon does not interact with the relaxing heavy hole's spin, as our data unambiguously demonstrate. Therefore the phonon does not carry with it any which-path information (apart from its energy), ${ }^{4}$ which would have rendered spectral filtering inefficient in restoring the entanglement. The reason is more involved and we discuss it below.

\section{DISCUSSION}

Figure 4(a) shows the cascade initiated by the ground state of a biexciton in an ideal, symmetric QD. In this case, the two exciton energy levels are degenerate, and emitted photon pairs will be entangled. ${ }^{1,5}$

Figure 4(b) shows the case of the ground-state biexciton cascade in an asymmetric QD in which the exciton levels are split by an energy $\delta$. Here, spectral filtering (cross-hatched rectangle) is necessary for the emitted photons to be entangled. ${ }^{4,11,12}$

Figure 4(c) shows a schematic plot of the two-photon probability distribution. The $x(y)$ axis represents the energy of the exciton (biexciton) photon. The dark-gray spots show the regions of high emission probability. Their size and shape are determined by the radiative width of the exciton $\left(\gamma_{X}\right)$ and biexciton $\left(\gamma_{X X}\right)$ lines. The emission in these regions is dominated by unentangled photon pairs. ${ }^{4,11,12}$ The energies of the two photons are related by total energy conservation: if the first photon has high energy, the second one will have low energy, and vice versa. This puts the two dark-gray spots on a line parallel to the $(1,-1)$ direction. The cross-hatched rectangle represents an optimal spectral filter for entanglement restoration. It is $\delta-\gamma_{X}$ by $\delta-\gamma_{X X}$ in size. It rejects most of the unentangled photon pairs while it keeps a measurable fraction of the entangled pairs, which lie mostly between the two dark-gray spots, on the connecting line. The degree of entanglement within the filtered photon pairs is thus (a)

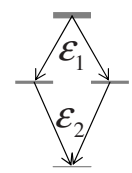

(d)

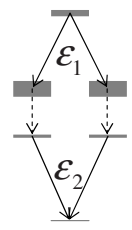

(b)

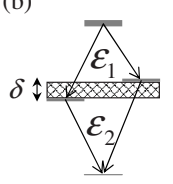

(e)

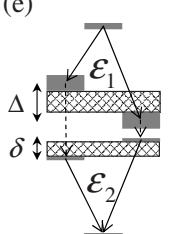

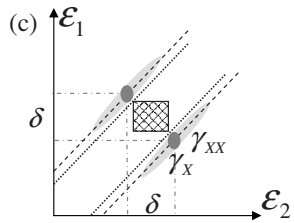

(f) $\uparrow \mathcal{E}_{1}$

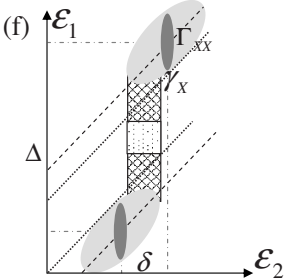

FIG. 4. (a) Ideal direct cascade. The widths of the levels represent their decay rate. (b) Ground-state biexciton cascade in an anisotropic QD. (c) Schematic two-photon probability distribution. Dark gray-high probability areas. Cross hatched-spectral filter. Light gray-inhomogeneous broadening due to spectral diffusion. [(d)-(f)] Same as (a)-(c) (respectively), for an indirect cascade. In (e) and (f) only the case of splittings in opposite directions is shown. The dotted rectangle in (f) is an example for a filter not penetrated by the high-probability areas for any amount of spectral diffusion.

increased. ${ }^{4,11,12}$ A narrower filter would yield higher degree of entanglement but it will transmit considerably less photon pairs.

Due to random fluctuations in the electrostatic environment of the QD, the energies of the spectral lines fluctuate with time. This "spectral diffusion" happens on time scales much longer than the radiative recombination time of the exciton. The random electric field is thus quasistatic. Since all spectral lines experience almost the same shift in a given static electric field, ${ }^{23}$ the energies of the exciton and biexciton lines will fluctuate in a correlated manner. The dark-gray spots of Fig. 4(c) will thus randomly move along the dashed lines parallel to the $(1,1)$ direction, as shown in the figure by the light-gray zones. As long as these areas are strictly outside the filtered area, spectral diffusion does not interfere with the entanglement restoration. Indeed, as was demonstrated in Ref. 4 and here [Fig. 3(a)], entanglement can be restored by spectral filtering for the ground biexciton (S) cascade.

The situation is somewhat different for the spin-blockaded cascade reported here. Figures 4(d)-4(f) describe this case. Here there is a fast, nonradiative (phononic) relaxation of the hole from its excited state to its ground state in between the biexciton and exciton radiative recombinations.

Since the spin of the exciton is conserved during the intermediate stage, and since the spatial parts of the exciton wave functions are identical for both decay paths, the emitted phonon does not carry any which-path information beyond its energy. One would therefore expect that appropriate filtering of the photon energies, and therefore also of the phonon energies, will restore the which path ambiguity, resulting in entanglement of the polarization state of the photons. We show below that for the present case, spectral diffusion prevents this from happening.

Figure 4(d) shows the ideal case where the excited and ground exciton states are each twofold degenerate. The short 
lifetime of the excited exciton states lead to a larger width $\left(\Gamma_{X X}\right)$ of the corresponding energy levels. Figure 4(e) shows the case of an asymmetric QD. The degeneracy is lifted for both ground and excited exciton states, albeit, as explained above, in opposite directions. Therefore, in one path both photons are more energetic while in the other path both are less energetic.

This is shown in Fig. 4(f), where the dark-gray spots again represent the regions of high probability. Their elongated shape is due to the larger width of the biexciton photon, which comes from the fast decay of its final state. Spectral diffusion will still shift these regions along the $(1,1)$ direction, as shown by light-gray zones. The analog of the optimal filter for this case is shown by the cross-hatched rectangle. It is $\Delta-\Gamma_{X X}$ by $\delta-\gamma_{X}$, where $\Delta$ is the excited exciton splitting (in absolute value). As in the previous case, such a filter excludes the dark-gray spots. However, it is not immune to spectral diffusion, as shown by the overlap of the cross-hatched rectangle and the light-gray zones. Indeed, as shown in Fig. 3(b), no entanglement could be detected even when this filter was applied.

Further decreasing the filter width may solve the spectral diffusion problem, as shown by the dotted rectangle in Fig. 4(f), which completely avoids the light-gray zones and any other possible location of the dark-gray spots. However, for such a filter the photon pair collection rate would be drastically lower. We note that if the ground and excited exciton states would have split to the same direction, the situation would have been similar to that described in Fig. 4(c), and entanglement restoration by spectral filtering would not have been affected by spectral diffusion, despite the phonon emission.

A quantitative condition for spectral filtering to effectively erase the which path information can be formulated by inspecting Figs. 4(c) and 4(f). Note first that spectral diffusion leads to motion of the dark-gray spots along the $(1,1)$ direction. Spectral filtering works if during this motion the spots stay strictly outside the filter area. The width of the filter that satisfies this condition is determined by looking at the projection of the filter on the orthogonal direction to the motion of the spots: the $(1,-1)$ direction. Let $f>0(F>0)$ be the filter width for the exciton (biexciton) photon spectral line. The filter's projection on the $(1,-1)$ direction is given by $(f+F) / \sqrt{2}$. The projection of the line connecting the centers of the two dark-gray spots on the $(1,-1)$ direction is given by $|\Delta \pm \delta| / \sqrt{2}$ where the plus sign is for Fig. 4(c) and the minus sign is for Fig. 4(f). For avoiding overlap one thus must have $F+f \leq|\Delta \pm \delta|$. The case of a minus sign forces narrow filter widths, which makes spectral filtering ineffective. Taking into account the widths of the lines (the sizes of the dark-gray spots), leads to a stronger constraint

$$
F+f \leq|\Delta \pm \delta|-\left(\Gamma_{X X}+\gamma_{X}\right)
$$

where $\Gamma_{X X}$ refers also to $\gamma_{X X}$ as appropriate. In this work, $\delta=34 \mu \mathrm{eV}, \Delta=140 \mu \mathrm{eV}, \gamma_{X}=1.5 \mu \mathrm{eV}, \gamma_{X X}=3 \mu \mathrm{eV}$, and
$\Gamma_{X X} \approx 40 \mu \mathrm{eV}$. Therefore, for the singlet-biexciton cascade, where $\Delta=\delta$, the constraint becomes $F+f \leq 2 \delta-\left(\gamma_{X X}+\gamma_{X}\right)$ $=63 \mu \mathrm{eV}$, just slightly smaller than $2 \delta=68 \mu \mathrm{eV}$. For the triplet-biexciton cascade we obtain $F+f \leq 64 \mu \mathrm{eV}$, This is now much smaller than $\Delta+\delta=174 \mu \mathrm{eV}$, and therefore hardly yields any coincidences. The dotted rectangle in Fig. 4(f) is an example of such a filter. For measuring the density matrix in Fig. 3(b) we used $f \approx 30 \mu \mathrm{eV}$ and $F \approx 100 \mu \mathrm{eV}$. We could not perform the experiment for a smaller $F$ due to diminishing coincidences signal. This explains why while entanglement was restored for the singlet-biexciton cascade, it was not restored for the triplet cascade.

\section{SUMMARY}

We identified optical transitions due to radiative decays of metastable, spin-blockaded hole-triplet-biexciton states in the PL spectrum of single neutral QDs. Photons emitted specifically from the symmetric two holes triplet state with zero spin projection $\left(\mathrm{T}_{0}\right)$ were found to be temporally correlated with photons emitted from the ground exciton transition $\left(X^{0}\right)$. In contrast to the correlations between the ground-state biexciton (in which the two holes form a spin singlet) and the exciton transitions, "bunching" (cascaded emission) in this case was observed for cross-linear polarizations. We explain this observation in terms of the spin-exchange symmetry of the initial, biexciton state: while the ground-state biexciton is antisymmetric under hole spin exchange, the triplet biexciton is symmetric. This reverses the polarization selection rules for the biexciton transitions.

The cascade involves an intermediate nonradiative relaxation. The measured correlations: bunching (antibunching) for cross-(co)linear polarizations show that this phononmediated relaxation preserves spin.

Two-photon polarization density matrices were measured for both cascades, with and without spectral filtering. Entanglement was restored for the ground biexciton cascade when spectral filtering was applied to it. Entanglement was not restored when spectral filtering was applied to the spinblockaded biexciton cascade. We attribute this deficiency of spectral filtering to the reversed energetic order of the excited exciton states. This reversed order allows unentangled photon pairs to penetrate the spectral filters during spectral diffusion of the emission lines.

\section{ACKNOWLEDGMENTS}

The support of the U.S.-Israel Binational Science Foundation (BSF), the Israeli Science Foundation (ISF), the Ministry of Science and Technology (MOST), and that of the Technion's RBNI are gratefully acknowledged. 
${ }^{1}$ O. Benson, C. Santori, M. Pelton, and Y. Yamamoto, Phys. Rev. Lett. 84, 2513 (2000).

${ }^{2}$ E. Moreau, I. Robert, L. Manin, V. Thierry-Mieg, J. M. Gérard, and I. Abram, Phys. Rev. Lett. 87, 183601 (2001).

${ }^{3}$ D. V. Regelman, U. Mizrahi, D. Gershoni, E. Ehrenfreund, W. V. Schoenfeld, and P. M. Petroff, Phys. Rev. Lett. 87, 257401 (2001).

${ }^{4}$ N. Akopian, N. H. Lindner, E. Poem, Y. Berlatzky, J. E. Avron, D. Gershoni, B. D. Gerardot, and P. M. Petroff, Phys. Rev. Lett. 96, 130501 (2006).

${ }^{5}$ R. Hafenbrak, S. M. Ulrich, P. Michler, L. Wang, A. Rastelli, and O. G. Schmidt, New J. Phys. 9, 315 (2007).

${ }^{6}$ C. L. Salter, R. M. Stevenson, I. Farrer, C. A. Nicoll, D. A. Ritchie, and A. J. Shields, Nature (London) 465, 594 (2010).

${ }^{7}$ A. Dousse, J. Suffczyński, A. Beveratos, O. Krebs, A. Lemaître, I. Sagnes, J. Bloch, P. Voisin, and P. Senellart, Nature (London) 466, 217 (2010).

${ }^{8}$ D. Gammon, E. S. Snow, B. V. Shanabrook, D. S. Katzer, and D. Park, Phys. Rev. Lett. 76, 3005 (1996).

${ }^{9}$ V. D. Kulakovskii, G. Bacher, R. Weigand, T. Kümmell, A. Forchel, E. Borovitskaya, K. Leonardi, and D. Hommel, Phys. Rev. Lett. 82, 1780 (1999).

${ }^{10}$ E. L. Ivchenko, and G. E. Pikus, Superlattices and Other Heterostructures: Symmetry and Optical Phenomena (Springer, New York, 1997).

${ }^{11}$ N. Akopian, N. H. Lindner, E. Poem, Y. Berlatzky, J. E. Avron, D. Gershoni, B. D. Gerardot, and P. M. Petroff, J. Appl. Phys. 101, 081712 (2007).
${ }^{12}$ E. A. Meirom, N. H. Lindner, Y. Berlatzky, E. Poem, N. Akopian, J. E. Avron, and D. Gershoni, Phys. Rev. A 77, 062310 (2008).

${ }^{13}$ E. Poem, Y. Kodriano, C. Tradonsky, B. D. Gerardot, P. M. Petroff, and D. Gershoni, Phys. Rev. B 81, 085306 (2010).

${ }^{14}$ E. Poem, J. Shemesh, I. Marderfeld, D. Galushko, N. Akopian, D. Gershoni, B. D. Gerardot, A. Badolato, and P. M. Petroff, Phys. Rev. B 76, 235304 (2007).

${ }^{15}$ T. Warming, E. Siebert, A. Schliwa, E. Stock, R. Zimmermann, and D. Bimberg, Phys. Rev. B 79, 125316 (2009).

${ }^{16}$ E. Poem, Y. Kodriano, C. Tradonsky, N. H. Lindner, B. D. Gerardot, P. M. Petroff, and D. Gershoni, Nat. Phys. (to be published).

${ }^{17}$ S. V. Gupalov, E. L. Ivchenko, and A. V. Kavokin, J. Exp. Theor. Phys. 86, 388 (1998).

${ }^{18}$ T. Takagahara, Phys. Rev. B 62, 16840 (2000).

${ }^{19}$ M. Ediger, G. Bester, B. D. Gerardot, A. Badolato, P. M. Petroff, K. Karrai, A. Zunger, and R. J. Warburton, Phys. Rev. Lett. 98, 036808 (2007).

${ }^{20}$ G. Bester, S. Nair, and A. Zunger, Phys. Rev. B 67, 161306(R) (2003)

${ }^{21}$ L. M. Woods, T. L. Reinecke, and R. Kotlyar, Phys. Rev. B 69, 125330 (2004).

${ }^{22}$ M. Trif, P. Simon, and D. Loss, Phys. Rev. Lett. 103, 106601 (2009).

${ }^{23}$ M. E. Ware, E. A. Stinaff, D. Gammon, M. F. Doty, A. S. Bracker, D. Gershoni, V. L. Korenev, Ş. C. Bădescu, Y. LyandaGeller, and T. L. Reinecke, Phys. Rev. Lett. 95, 177403 (2005). 